\title{
Review: exploration of psychological and physical health differences between caregivers and non-caregivers
}

Pinquart $M$, Sörensen S. Differences between caregivers and noncaregivers in psychological health and physical health: a meta-analysis. Psychol Aging 2003;18:250-67.

What are the differences between caregivers and non-caregivers in perceived stress, depression, subjective well being, physical health and self efficacy?

METHODS

-

Design: Systematic review with meta-analysis.

Data sources: MEDLINE, PsycINFO, Psyndex (search dates not stated). Reference lists were hand searched.
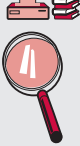

Study selection and analysis: Eligible studies compared levels of perceived stress, depression, general subjective well being, physical health, or self-efficacy between a sample of informa caregivers of older adults and a sample of non-caregivers. Exclusions: studies where reported differences between caregivers and non-caregivers could not be converted to standard deviation units (see notes); not written in English, French, German, or Russian.

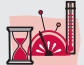

Outcomes: Sociodemographic differences, the impairment of the care receiver, and effect sizes (derived for each study either from the difference in outcome between groups divided by the pooled standard deviation; or from reported significance values; or using a "vote counts" methodology) of perceived stress, depression, subjective well being, physical health, and self efficacy.

\section{MAIN RESULTS}

Eighty four studies met inclusion criteria (number of participants not stated). There was substantial heterogeneity among studies (see Notes). Approximate ranges in effect sizes between caregivers and non-caregivers (where positive values represent larger impairments of caregivers compared with non-caregivers) were -0.5 to 3.5 for perceived stress ( 29 studies); -0.25 to 1.7 for depression ( 78 studies); -1.5 to 0.25 for subjective well being (46 studies); -0.8 to 0.4 for physical health (64 studies), and -1.4 to 0 for self efficacy. Metaanalysis found that caregivers had significantly higher levels of stress and depression and significantly lower levels of subjective well being, physical health, and self efficacy than non-caregivers.

\section{CONCLUSIONS}

The authors conclude that caregivers had higher levels of stress and depression and were more likely to have lower levels of subjective well being, physical health, and self efficacy than non-caregivers.

For correspondence: Martin Pinquart Department of Developmental Psychology, Friedrich Schiller University, Jena, Germany; Martin.Pinquarł@ rz.uni-jena.de

Sources of funding: not specified.

\section{NOTES}

The systematic review did not exclude studies based on their design or quality of their methods. It is not surprising, therefore, to find heterogeneity of results. Under these circumstances, narrative synthesis may be preferable to a meta-analysis. Excluding studies on the basis of the outcome measure used may result in important studies being excluded from the analysis. This may affect the reliability of the results. The majority of studies were focused on caregivers of demented elderly, which may reduce the generaliseability of the results.

\section{Commentary}

The shift towards minimising hospitalisation has led to a greater role for non-professional carers. A common belief is that for some conditions and for some families this can be a stressful experience, which strains their coping resources. This study critically examines this belief.

The interpretation of studies on carers can be problematic for several reasons; the samples may be non-representative and biased towards those with more problems, namely those presenting to services for help. On the other hand, data ascertained from community samples may not accurately define the amount of care provided. Furthermore the comparison group may not be adequately matched on demographic features etc. Also, publication bias may inflate carers' stress, in that only studies which show differences between caregivers and non-caregivers get published. These factors were taken into account within this systematic review.

The results are somewhat surprising as only $8 \%$ of the variance in carer ill health was accounted for by care giving. Thus if we interpret these findings within the general stress coping model which has often been applied to the care giving experiences eg Lazarus and Folkman's cognitive stress theory ${ }^{9}$ then it is clear that most care givers are resiliant and have effective coping strategies. However we cannot be certain that these findings from older adults can be generalised. For example, the difference in the base rate of psychological and physical distress and the additional roles and expectations of the caregivers for young people with severe illness may mean that the proportionate burden caused by care giving may differ. Nevertheless this may change the balance in research on carers and cause us to look to the people who cope well in order to find strategies to increase the competencies of those carers who have more difficulties.

Professor Janet Treasure GKT Medical School \& Institute of Psychiatry, Kings College, London UK 1 Lazarus RS, Folkman S. Stress appraisal and coping. New York: Springer Publishing Company, 1984. 